\title{
Sand/cement ratio evaluation on mortar using neural network and ultrasonic transmission inspection
}

\author{
M. Molero ${ }^{1,2}$, M.G. Hernández², M.A.G. Izquierdo ${ }^{1}$, J.V. Fuente ${ }^{3}$, J.J Anaya ${ }^{2}$ \\ ${ }^{1}$ Departamento de Señales, Sistemas y Radiocomunicaciones, ETSI Telecomunicación (UPM), Spain, molero@iai.csic.es \\ 2 Instituto de Automática Industrial (CSIC), La Poveda 28500 Arganda del Rey, Spain \\ ${ }^{3}$ Instituto Tecnológico de la Construcción (AIDICO), Parc tecnològic 46980 Paterna, Valencia, Spain
}

\begin{abstract}
The quality and degradation state of building materials can be determined by means of nondestructive testing (NDT). These materials are composed by a cementitious matrix and several aggregates, generally sand and gravel. Sand/cement ratio (s/c) provides the final material quality; however, the sand content can mask the matrix properties in a nondestructive measurement.

This work presents an ultrasonic evaluation of the mortar sand content. This evaluation is carried out with several mortar probes which have been varied both, in the quality of the cementitious matrix, and in the s/c ratio. This ratio has been determined by the ultrasonic transmission inspection information with different transducers. Statistical principal component analysis (PCA) in order to reduce the dimension of the captured traces is applied. Feed-forward neural networks have been trained using a reduced PCA and the outputs of the network allow displaying a false color map that shows the mortar probes (s/c) distribution.
\end{abstract}

Key words: Ultrasonic signal processing, material characterization, NDT, neural network application.

\section{A. Introduction}

Cement is an inorganic material that mixed with water has the property to set and to harden even under water. By virtue of chemical reactions during the hydration maintains high resistance and stability. When cement is mixed with water and fine aggregates (sand), it generates mortar, and when it is mixed with fine aggregates and coarse aggregates (gravel, crushed stones) generates concrete. On the characterization of the cementitious materials a much studied parameter is the compressive strength (macroscopic level), nevertheless, when the research is oriented to the deterioration of these materials, it is necessary a micro-structure analysis. Mortar micro-structure is characterized, among other parameters, by the type of cement, the water/cement ratio (w/c) and sand/cement ratio (s/c). Although, in the manufacture of mortar these relations come fixed a priori, during the setting and hardening process, the sand decants due to the difference of densities with respect to the cement paste.

Neural networks have been widely used for the evaluation, characterization and prediction of parameters in cementitious materials. Diverse authors predict the compressive strength from supervised neural networks [1]-[3] or artificial intelligence techniques [4], [5] using parameters as: (w/c), the fine and coarse aggregates, densities, mass proportions, among other properties of these materials (i.e. ash, additives). Ji et al [6] use neural networks to develop a design strategy for selecting the optimal parameters values in the concrete manufacturing. Kewalramani et al [7] use neural networks to relate the speed of an ultrasonic pulse with the compression strength in mortar and concrete samples. On the other hand, few applications on principal components analysis (PCA) of ultrasonic transmission signals are found in the literature. Carlson and Martisson [8] have used the PCA of the ultrasonic transmission signal for the identification of gas mixtures.

However, few works with neural networks and PCA have been carried out with ultrasonic micro-structural evaluation on mortar and concrete. The goal of this work is the use of neural networks combined with PCA for the ultrasonic evaluation of the sand/cement ratioWorking on the manuscript

\section{B. Theory}

\section{B.1. Principal Components Analysis}

One of the main problems in the multivariate analysis of data is the dimensions reduction. Let us suppose that the original data consists of $n$ observations of dimension $p$, it implies that the data matrix $\boldsymbol{X}$ is $n \times p$ and the covariance matrix $\boldsymbol{S}$ is $p \times p$. The objective of the principal components analysis (PCA) is to describe each observation by means of a new vector of dimension $q$ (where $q<p$ ) called principal component (PC). These components can be obtained by defining a linear transform which maximizes the data variance. The first PC, $z_{1}=\boldsymbol{X} a_{1}$ is obtained projecting the data in the direction of the unitary vector $a_{1}$ in which the variance of the projected data $z_{1}$ is maximum. The second PC is obtained projecting the data in the unitary direction $a_{2}$ that maximizes $a_{2}^{T} \boldsymbol{S} a_{2}$ between those directions that are orthogonal with $a_{1}$. To summarize, the $z_{i}$ is obtained projecting the data in the unitary direction $a_{i}$ that maximizes $\boldsymbol{a}_{i}^{T} \boldsymbol{S} a_{i}$ between those directions that are orthogonal to $a_{1}, \ldots, a_{i-1}$; the key is to considerate successive projections throughout the possible "most different” direction. 


\section{B.2. Artificial Neural Networks Architecture}

Neural networks have been a modeling scheme widely used for the multivariate non-linear analysis. The optimal nature and easy implantation of neural networks has been the main reasons for its use in different knowledge fields. A supervised feed-forward neural network finds a function $y_{j}=f\left(x_{i}\right)$ where $x_{i}$ is the input data, $y_{j}$ is the output class, and $f(\bullet)$ is the non-linear function that relate the input vector with the output class. When a neural network is trained with desired outputs $d_{j}$, for a limited input set $x_{i}$, the main problem is to find the weight values on the estimation process $\left\{\omega_{i j}\right\}$ that better fit to the function $f(\bullet)$ for all pairs $\left(x_{i}, y_{j}\right)$, even for pairs that have not been used in the training process.

\section{Experimental Data}

\section{C.1. Material Processing (the probes)}

Twenty-eight mortar probes which have been varied both, in the cement quality, and in the s/c ratio, have been inspected by an ultrasonic transmission system. Table 1 shows the properties of each probe which are divided in two sets. These sets correspond to kinds of cement: a) CEM IV/B-L 32.5N and b) CEM II/A-L 42.5R, both with different water/cement ratios. According to this table, manufacture water/cement ratio considerably varies between the two sets. Two important aspects about those probe sets have to be considered; first, all probes were made at the same time except the probes corresponding to the sand/cement ratio 2.0 and 3.0, and also the second probe set are fractured since these have been used for experiments and measurements for destructive testing. The original size of the probes is $40 \times 40 \times 160$ [mm].

Table 1. Properties of the two mortar probe sets. Second and third column show the probes for each s/c ratio.

\begin{tabular}{|c|c|c|}
\hline s/c & w/c (set 1) & w/c (set 2) \\
\hline 0.0 & $0.4,0.5$ & $0.315,0.365$ \\
\hline 0.5 & $0.4,0.5$ & $0.338,0.388$ \\
\hline 1.0 & $0.4,0.5$ & $0.360,0.410$ \\
\hline 1.5 & $0.4,0.5$ & $0.383,0.433$ \\
\hline 2.0 & $0.4,0.5$ & $0.450,0.500$ \\
\hline 2.5 & $0.4,0.4$ & $0.428,0.474$ \\
\hline 3.0 & 0.5 .0 .5 & $0.450,0.500$ \\
\hline
\end{tabular}

\section{C.2. Ultrasonic Inspection}

Ultrasonic inspection of mortar probes has been carried out using an automatic system that consists on: i) a pool, where probes are submerged in water for the inspection, ii) an automatic positioning system for $(x, y, z)$ coordinates, working with step motors, iii) an ultrasonic generator and acquiring system and iv) a specialized software for processing and storage the signals. Inspection has been made using two ultrasonic transducers with a transmission configuration. When the transducers are aligned, the signal in the receiver is a function of the sample structure in that zone. If it is free of heterogeneities, the amplitude will be maxima whereas it will be diminishing as the beam goes through discontinuities of increasing size. By each mortar probe, two inspections have been made: i) with a $1 \mathrm{MHz}$ KH1N transducer, and ii) using a transducer of $2 \mathrm{MHz} \mathrm{KH} 2 \mathrm{~K}$. The acquiring system has made a spatial averaging considering four signals, separated in distance $0.1 \mathrm{~mm}$. It stores an average of $n=2080$ signals with $p=412$ samples for each probe (49920 $\times 412$ signals in total), using a sampling frequency of $40 \mathrm{MHz}$.

\section{C.3. Data Processing}

Several mortar probes are evaluated and characterized based on their manufacture $(\mathrm{s} / \mathrm{c})$ ratio. The procedure that generate a final $(\mathrm{s} / \mathrm{c})$ characterization image is showed in Fig 1. This procedure has five stages:

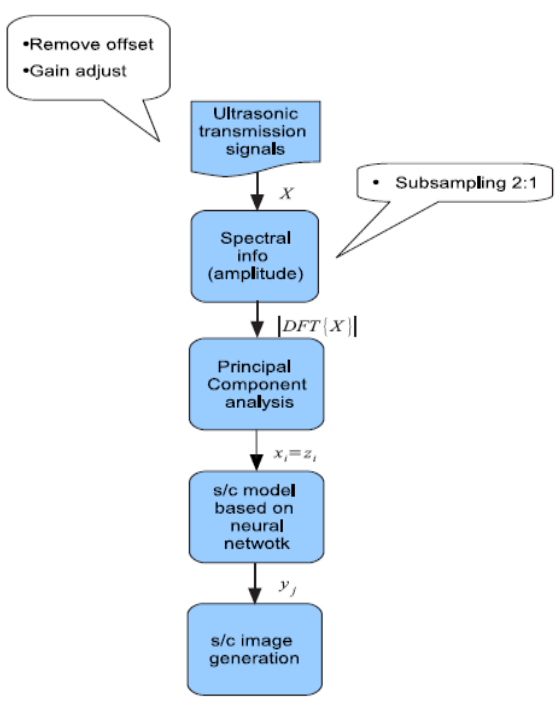

Fig. 1. Procedure diagram of data processing.

- Pre-processing of the ultrasonic signals: This stage consists in: i) a subsampling 2:1, and ii) a gain adjusts.

- DFT magnitude computation.

- Principal components analysis: A transformation matrix is obtained for each transducer frequency. Therefore, a set of basis functions is defined corresponding to the pulse deformation.

- Neural network model estimation: Principal components from signals at $1 \mathrm{MHz}$ and $2 \mathrm{MHz}$ are used for training a supervised a two hidden-layer feed-forward neural network. The inputs can be expressed as $y_{j}=\left[\begin{array}{lll}c_{1} & c_{2} \ldots c_{j}\end{array}\right]$, where $c_{j}$ is a component that represents one class which will have a value near one if the signals are of this class, otherwise a value near zero. The classes are sand dependent.

- (s/c) image generation: By means of the output vectors, false color images are generated. These images represent the $(\mathrm{s} / \mathrm{c})$ distribution on mortar probes.

As result, the neural network classifies the signals according to the sand proportion by its corresponding spectral magnitude. Using only the spectral magnitude 
information of the transmission signals, this evaluation does not consider the phase data or the velocity information of ultrasonic signals.

\section{Results and Discussion}

According to the previous section, a (s/c) characterization image, from a model defined by a neuronal network, is generated. We have considered two experiments according to the two sets of mortar probes with cement types: i) separately and ii) jointly. In both experiments, we only consider the following classes according to the $(\mathrm{s} / \mathrm{c})$ ratio of $0,0.5,1.0,1.5$ and 2.5 for the training network process.

The characteristic of the experiments are:

- In the first experiment, 84 signals of each probe are used for the neural network training process. As result, a matrix $\boldsymbol{X}$ with $n=420$ signals is defined that includes mentioned sand/cement ratio for each transducer frequency. In this experiment, we have two cases that depend of the cement type:

a) CEM IV/B-L $32.5 \mathrm{~N}$ : In the PCA stage, we have used six $z_{i}$ at $1 \mathrm{MHz}\left(q_{1}=6\right)$ and the two $z_{i}$ at $2 \mathrm{MHz}\left(q_{2}=2\right)$. The architecture of this network has been chosen as $8 / 40 / 40 / 5$.

b) CEM II/A-L 42.5R: with $q_{1}=7$ and $q_{2}=3$. The network architecture was defined as 10/50/50/5.

- In the second experiment, apart of varying the sand/cement ratio, and also considers the two cement types. This was made to verify that the ultrasonic signals not only are affected by the sand content, but also by the different cement matrixes [9]. The network is trained using 84 signals with $p=412$ samples corresponding to each one of the mortar probes, with a total of 420 signals for each cement type. Therefore, the matrix contains $n=840$ signals. In this experiment, we have trained the neural networks using the two cement type at the same time:

a) CEM IV/B-L 32.5N and CEM II/A-L 42.5R: The architecture was chosen as 10/50/50/5 with $q_{1}=7$ and $q_{2}=3$.

Notice that the ultrasonic pulse undergoes deformations when traveling through the probe. The 2 $\mathrm{MHz}$ transducer signals are more prone to such deformation as shown in Fig. 2. However, when the sand content is increased in the cement matrix, we observe a greater pulse deformation in both 1 and $2 \mathrm{MHz}$ spectra (Fig. 2d). Nevertheless, the extraction of the information via $z_{i}$ makes more emphasis in the form of the pulse that in the spectrum central frequency. Fig. 3 and 4 show the (s/c) image for each cement type corresponding to the $1^{\text {st }}$ and $2^{\text {nd }}$ experiment, respectively. Each two probes correspond to the classes: 0, 0.5, 1.0, 1.5 and 2.5.
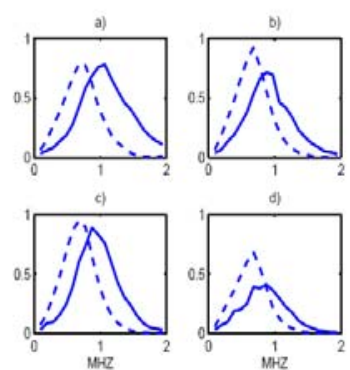

Fig. 2. Spectra of the received transmission pulse at $1 \mathrm{MHz}$ (dot line) and $2 \mathrm{MHz}$ (solid line) for the 1st experiment with different s/c ratio classes: a) 0 , b) 0.5 , c) 1.0 , d) 2.5 .
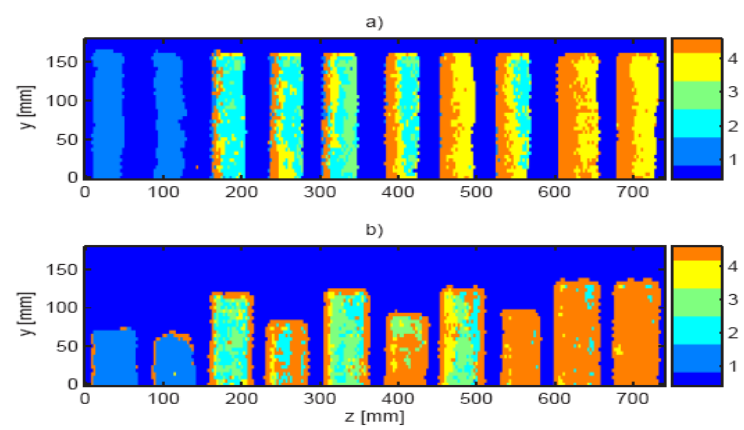

Fig 3. (s/c) image of mortar probes of $1^{\text {st }}$ experiment (separately training): first probe set and $b$ ) second probe set.
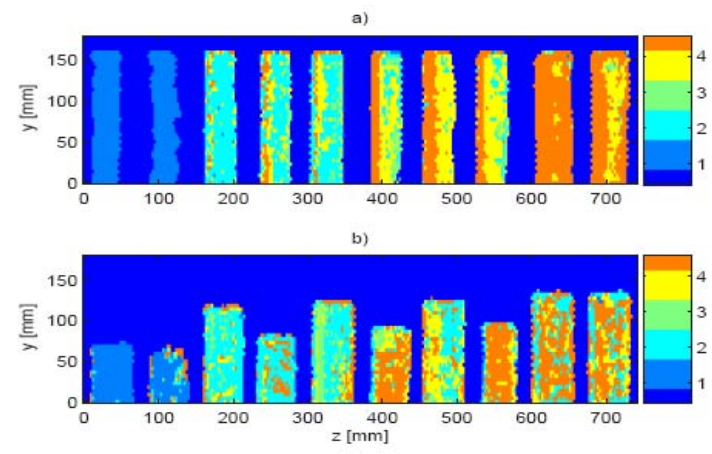

Fig 4. (s/c) image of mortar probes of $2^{\text {nd }}$ experiment (separately training): first probe set and b) second probe set.

The images allow displaying a false color map that shows the estimated (s/c) ratio. In addition, we can estimate the average $(\mathrm{s} / \mathrm{c})$ ratio for each probe from such images. As a result, a final estimation of the sand content is obtained. Indeed, these results provide an idea of the geometric sand distribution inside the probes. The values of the average $(\mathrm{s} / \mathrm{c})$ ratio for each experiment using the two probe sets are shown in Figure 5. These results correspond to: 1) desired distribution, 2) 1st experiment using the first probe set, 3) 1st experiment using the second probe set, 4) 2nd experiment using the first probe set, and 5) 2nd experiment using the second probe set. Sand content characterization is possible from these results. However, extreme values are easier estimated than the middles. It is also observe that when information of both cement types is included, greater difficulties for the evaluation exist. However, the distribution trends stay between the manufacture $(\mathrm{s} / \mathrm{c})$ ratios and the estimated ratios. 


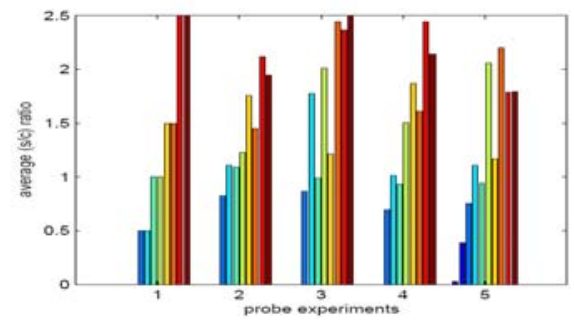

Fig. 5. Distribution of the average ratio for each probe.

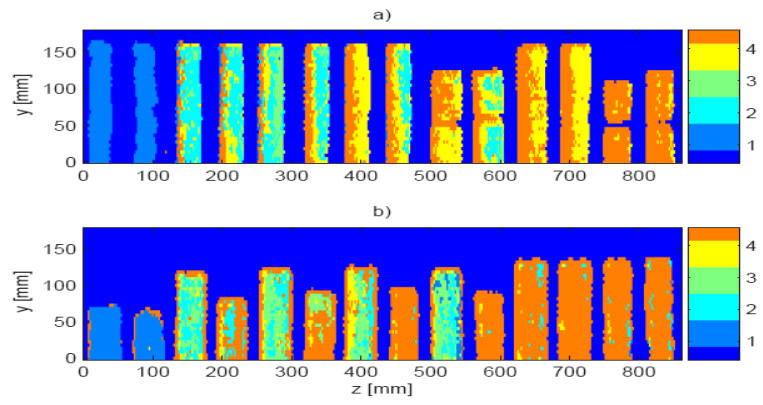

Fig 6. (s/c) image of mortar probes of $1^{\text {st }}$ experiment (jointly training): first probe set and b) second probe set.

Figures 6 and 7 present the (s/c) characterization images from others sand/cement ratios which have not used in the training process. Those probes are: the ninth and the tenth corresponding to the ratio 2.0; the thirteenth and the fourteenth corresponding to the ratio 3.0. We note that for the first pair of probes, the neural network model can not estimate the right class, whereas for the second pair; the estimation produce the expected value.

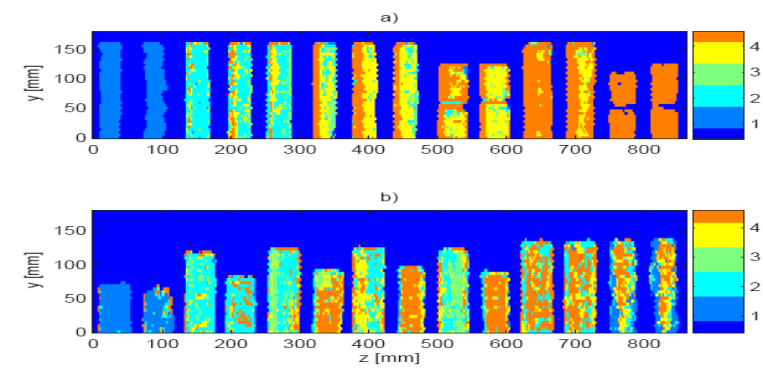

Fig 7. (s/c) image of mortar probes of $2^{\text {nd }}$ experiment (jointly training): first probe set and b) second probe set

\section{E. Conclusions}

In order to evaluate the sand/cement ratio of mortar probes, we have used principal components analysis (PCA) from frequency information because is less sensitive to the pulse deformation due to the sand grains and the cement matrix scattering effects. Several Neural networks have been trained using these principal components and their outputs have allowed displaying a false color map that shows the distribution and the average of the $(\mathrm{s} / \mathrm{c})$ ratio of the mortar probes. We have observed in these images the non-homogenous distributions of the sand inside the probes. This work has shown that the $(\mathrm{s} / \mathrm{c})$ ratio of the mixture of manufactured cement paste and sand may change due to the setting process.

\section{F. Acknowledgment}

The financial support of Spanish Ministry of Education and Science (Project BIA 2006-15188-C0301) and Spanish Ministry of Public Works (C14/2006) is acknowledged. M Molero is supported by the Programme Alban, the European Union programme of high level Scholarchip for Latin America (E05D055458MX) and The Mexican National Council for Science and Technology Conacyt: (186384). M.G. Hernández is supported by a postdoctoral CSIC-I3P contract granted by the European Social Fund.

\section{G. Literature}

[1] I. Yeh. Modeling of strength of high-performance concrete using artificial neural networks. Cement and Concrete Research, 28(2):1797-1808, 1998.

[2] N. Hong-Guang and W. Ji-Zong. Prediction of compressive strength of concrete by neural networks. Cement and Concrete Research, 30:1245-1250, 2000.

[3] Seung-Chang Lee. Prediction of concrete strength using artificial neural networks. Engineering Structures, 25:849-857, 2003.

[4] S. Akkurt, S. Ozdemir, G. Tayfur, and B. Akyol. The use of ga-anns in the modelling of compressive strength of cement mortar. Cement and Concrete Research, 33:973979, 2003.

[5] S.D. Kim, D.H. Shin, L.M. Lim, J. Lee, and S.H. Kim. Design strength identification of concrete by ultrasonic signal processing based on artificial intelligence techniques. IEEE Trans. on UFFC, 52(7):1145-1151, 2005.

[6] T. Ji, T. Lin, and X. Lin. A concrete mix proportion design algorithm based on artificial neural networks. Cement and Concrete Research, 36:1399-1408, 2006.

[7] M. Kewalramani and R. Gupta. Concrete compressive strength prediction using ultrasonic pulse velocity through artificial neural networks. Automation in Construction, 15:374-379, 2006.

[8] J. Carlson and P. Martinsson. Exploring interaction effects in two-component gas mixtures using orthogonal signal correction of ultrasound pulses. Journal of Acoustical Society of America, 117(5):2961-2968, 2005.

[9] M.G. Hernández, J.J. Anaya, L.G. Ullate, and A.Ibañez. Formulation of a new micromechanic model of three phases for ultrasonic characterization of cement-based materials. Cementand Concrete Research, 36:609-616, 2006. 